\title{
Optical investigations of the electrodynamics of $\mathrm{UPd}_{2} \mathrm{Al}_{3}$
}

\author{
M. Dressel ${ }^{a *}$, L. Degiorgi ${ }^{b}$, G. Grüner ${ }^{a}$, P. Wachter ${ }^{b}$, N. Sato ${ }^{c}$, T. Komatsubara ${ }^{c}$, \\ Y. Uemura ${ }^{\mathrm{d}}$ \\ 'Department of Physics and Solid State Science Center. University of California, Los Angeles, CA 90024-1547. USA \\ 'Laboratorium für Festkörperphysik, Eidgenössische Technische Hochsschule, CH-8093 Zürich. Switzerland \\ 'Department of Physics. Faculty of Science. Tohoku University, Sendai 980. Japan \\ 'Deparment of Phisics, Columbia Unilersity, New York, NY 10027, USA
}

\begin{abstract}
We have investigated the electrodynamic response of $\mathrm{UPd}_{2} \mathrm{Al}_{3}$. At low temperatures, we observe the formation of a low-frequency narrow resonance, which indicates the development of the many-body coherent state. We do not find any evidence of a gap absorption associated to a spin density wave state below the antiferromagnetic phase transition at $T_{\mathrm{N}}=14 \mathrm{~K}$, which would develop as a consequence of a Fermi surface instability.
\end{abstract}

The heavy Fermion superconductor $\mathrm{UPd}_{2} \mathrm{Al}_{3}$ is one of the fascinating materials, besides $\mathrm{URu}_{2} \mathrm{Si}_{2}$ and $\mathrm{UNi}_{2} \mathrm{Al}_{3}$ $[1,2]$, showing the coexistence of both superconductivity and magnetic ordering [3]. The de magnetic susceptibility exhibits a kink at $T_{\mathrm{N}}=14 \mathrm{~K}$ and a remarkable drop at about $T_{c}^{\prime}=2 \mathrm{~K}$, where also the specific heat shows clear anomalies.

The DC resistivity $\rho(T)$ shown in Fig. 1 bears a close similarity with the transport properties of other heavy Fermions. Above the broad maximum at $T_{\text {co }}=80 \mathrm{~K}$. $\rho(T)$ decreases with increasing temperature in a manner similar to that obseried in metals containing isolated magnetic impurities, suggesting a Kondo scattering mechanism. Below the coherence tempera:ure $T_{\text {cos }}$ where the many-body effects progressively develop, there is first the antiferromagnetic transition at $T_{\mathrm{N}}=15 \mathrm{~K}$. which reflects the freezing out of spin disorder scattering. and then a sharp drop io zero at the superconducting transition $T_{1}=1.7 \mathrm{~K}$. While in $U R u_{2} \mathrm{~S}_{2}$ the antifer-

* Corresponding author.

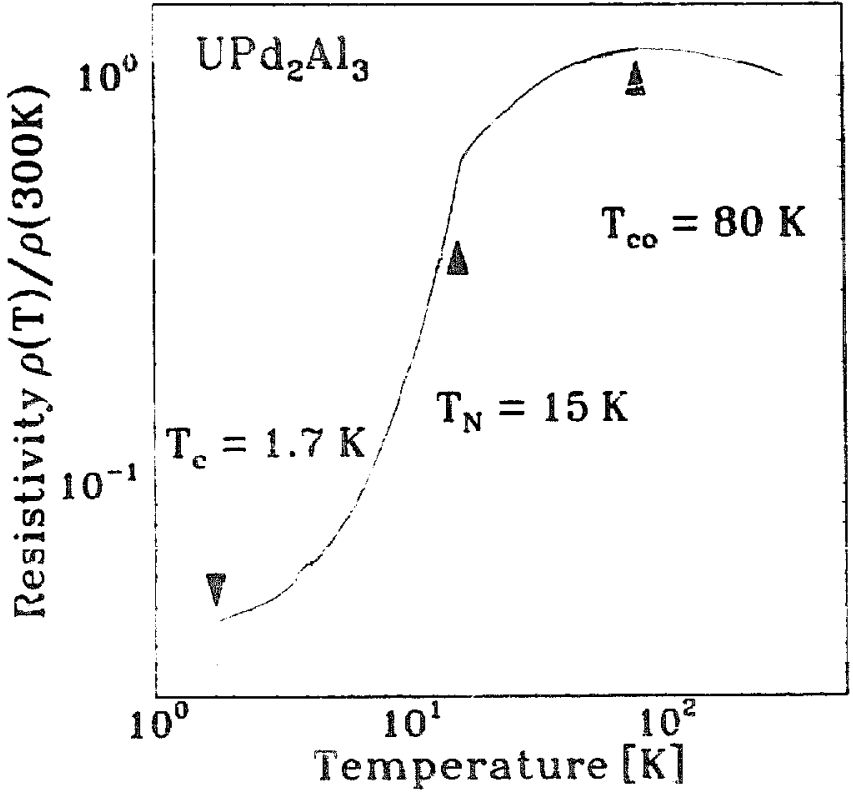

Fig. 1. Temperature dependence of the electrical resistivity of I $\mathrm{Pd}_{2} \mathrm{Al}_{3}$ normalized in the room temperature balue. 


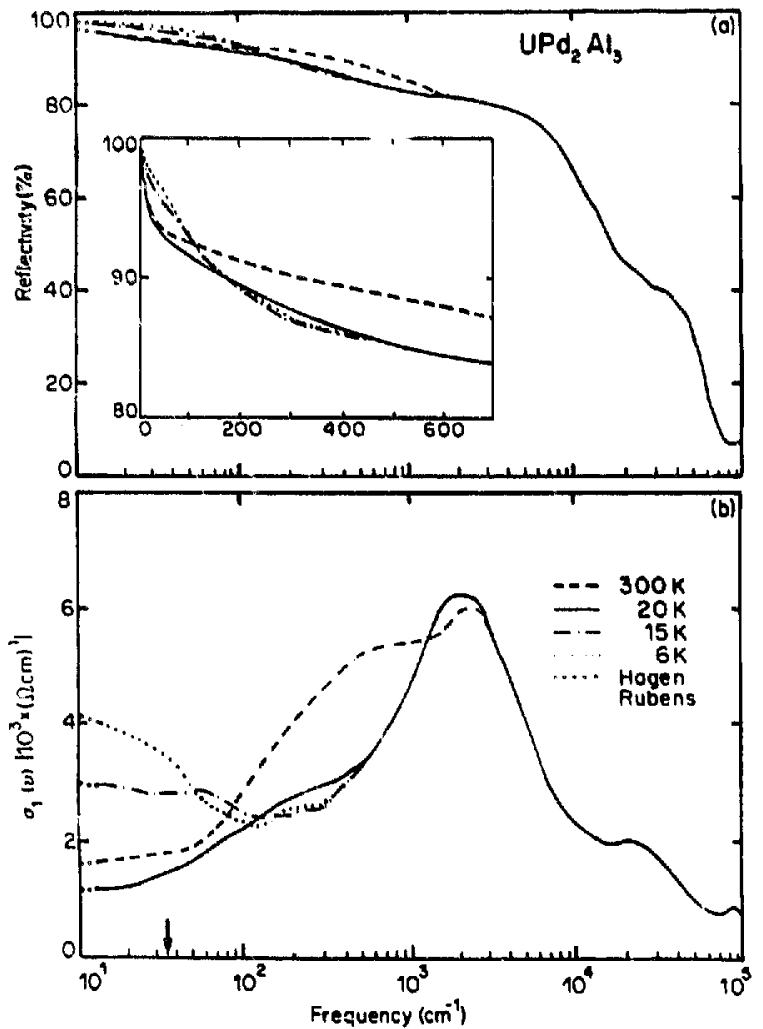

Fig. 2. (a) Reflectivity of UPd $\mathrm{Al}_{3}$ at several temperatures in the entirs frequency range. The inset displays the far infrared range of the reflectivity on an expanded scale. (b) Optical conductivity obtained through Kramers Kronig transformation of the reflectance spectra. The arrow marks the expected position of the SDW gap $2.1=3.53 \mathrm{~d} k_{\mathrm{n}} T_{\mathrm{N}}$

Table 1

Parameters of the renormalized Drude fit of the optical conductivity at different temperatures

\begin{tabular}{llll}
\hline & $\begin{array}{l}\text { Plasma } \\
\text { frequency } \\
h_{\mathrm{p}}(\mathrm{eV})\end{array}$ & $\begin{array}{l}\text { Damping } \\
\Gamma_{\mathrm{b}}(\mathrm{eV})\end{array}$ & $\begin{array}{l}\text { Effective mass } \\
m^{*} m_{\mathrm{b}}\end{array}$ \\
\hline$T=300 \mathrm{~K}>T_{\text {co }}$ & 4 & 0.025 & 1 \\
$T=15 \mathrm{~K}<T_{c}$ & 0.754 & 0.025 & 97 \\
$T=6 \mathrm{~K}<T_{\checkmark}$ & 0.47 & 0.007 & 67 \\
\hline
\end{tabular}

romagnetic-like phase transition at $17.5 \mathrm{~K}$ shows up in a well-defined anomaly similar to the one at the spin density wave ( $S D W$ ) transition in $\mathrm{Cr}$, in $\mathrm{UPd} \mathrm{Pd}_{2} \mathrm{Al}_{3}$ we find only a change in slope of $\rho(T)$. The SDW-like transition in $\mathrm{URu}_{2} \mathrm{Si}_{2}$ corresponds to a nesting at $2 k_{1}$ of the formi surface which directly affects the electronic density of states. Consequently, a transition across the single particle gap has been found in the excitation spectrum [4].

Besides the characterization of the samples by X-ray defraction, magnetization, d.c. and microwave resistivity experiments, we have investigated the optical reflectance on a broad frequency range between 14 and $10^{5} \mathrm{~cm}^{-1}$ at different temperatures $(6-300 \mathrm{~K})$. Figure $2(\mathrm{a})$ displays the optical reflectivity $R(v)$ at several significant temperatures above and below $T_{\mathrm{cc}}$ and $T_{\mathrm{N}}$, as well. The inset is a blowup of the far infrared frequency range. The optical conductivity $\sigma_{1}(v)$ is obtained through a Kramers-Kronig transformation of $R(v)$ and shown in Fig. 2(b). Details are published elsewhere [5]. At $300 \mathrm{~K}$ we see a frequencyindependent response at low frequencies and several absorptions at high frequencies due to interband transitions. At low temperatures in accordance with the crossover to higher reflectivity in the far infrared, we observe the formation of a temperature-dependent narrow Drude-like response.

in contrast to optical results on $\mathrm{URu}_{2} \mathrm{Si}_{2}$ [4], no absorption was found around $2 \mathrm{~A}=3.53 k_{\mathrm{B}} T_{\mathrm{N}}=$ $35 \mathrm{~cm}^{-1}$. This is, however, expected since the antiferromagnetic transition is commensurate with the lattice and it is not the consequence of the $2 k_{\mathrm{F}}$ nesting of the Fermi surface. Therefore, no SDW-gap opens at the Fermi level.

The only temperature dependence in $\sigma_{1}(v)$ is related to the temperature-dependent Drude-like resonance in the far infrared (Fig. 2(b)), ascribed to the optical conductivity of the heavy quasiparticle. The narrow resonance can be fitted with a renormalized Drude expression $[6,7]$ :

$\sigma(\omega))=\frac{n e^{2} \tau^{*}}{m^{*}} \frac{1}{1-\mathrm{i} \omega \tau^{*}}$

where $m^{*}$ and $\tau^{*}$ are effective mass of the heavy quasiparticles and the renormalized scattering time, respectively. The parameters of the fit at different temperatures are listed in Table 1. Our results are in good agreement with estimations applying the Pippard formula [5], where the known values of the mean frie path, the coherence length and the penetration depth are used [3]. We are also in accord with specific hear measurements and obtain the enhancement of the effective mass $m^{*}=68 m_{\mathrm{r}}$ and the total charge currier concentration $n_{\mathrm{c}}=1.0910^{22} \mathrm{~cm}^{3}$.

In conclusion, we have measured the complete electrodynamic response of $\mathrm{UPd}_{2} \mathrm{Al}_{3}$ at temperatures above and below $T_{\text {an }}$ and $T_{\mathrm{N}}$. We do not find evidence of an absorption associated to a SDW energy gap, in accordance with the commensurate nature of the antiferromagnetic transtion. At how temperatures, we do find a narrow resonance centered at $p=0$ and can describe it with a renormalized Drude formalia. We have extracted 
several intrinsic parameters characterizing this coherent many-body state, and found that the enhancement of the effective mass of the heavy quasiparticle is in perfect agreement with the thermodynamic results.

\section{Acknowledgements}

We acknowledge the help of S. Bruder, O. Klein and J. Müller. We are also grateful to T.M. Rice and H.R. Ott for valuable discussions. Research at UCLA was supported by the NSF grant DMR 92-16500. One of us (M.D.) acknowledges support of the Alexander von Humboldt-Foundation.

\section{References}

[1] M.B. Miaple, JW. Chen, Y. Dalichaouch, T. Koheia, $C$. Rossel and M.S. Torikachvili, Phys. Rev. Lett. 56(1986) 185.
[2] C. Geibel, S. Thies, D. Kaczorowski, A. Mehner, A. Gratud. B. Seidel, U. Ahtheim. R. Helfrich. K. Petersen. C.D Bredl and F. Steglich, Z. Phys. B 83 (1991) 305.

[3] C. Geibel, C. Schank, S. Thics, H. Kitaziwil, C.D. Bredl. A. Boehm. M.Rau, A. Grauel, R. Caspary. R. Helfrich. U. Ahlheim, G. Weber and F. Steglich, Z. Phys. B 84 (1991) 1.

[4] D.A. Bonn, j.D. Garrett and T. Timusk, Phys. Rev. Leti. 61 (1988) 1305.

[5] L. Degiorgi, M. Dressel, G. Grüner, P. Wachter, N. Sato and T. Komatsubara, submitted for publication in Europhys. Lett.

[6] I.B. Ferreria, A.M. Awasthi. L. Degiorģi, G. Grüner. Y. Dalichaouch and M.B. Maple, Solid State Commun. 83 (1992) 27.

[7] A.M. Awasthi, L. Degırgi, G. Grüner, Y. Dalichaouch and M.B. Maple, Phys. Rev. B 48 (1993) 10692. 